\title{
On the maximum principle for elliptic operators in weighted spaces
}

\section{Loredana Caso* and Roberta D'Ambrosio}

"Correspondence: Iorcaso@unisa.it Dipartimento di Matematica, Università di Salerno, Via Giovanni Paolo II, I - 84084, Fisciano, Italy

\begin{abstract}
We establish a maximum principle for subsolutions of second order elliptic equations. In particular, we consider some linear operators with leading coefficients locally VMO, while the other coefficients and the boundary conditions involve a suitable weight function.
\end{abstract}

MSC: $35 \mathrm{~J} 25 ; 35 \mathrm{R} 05 ; 35 \mathrm{~B} 50$

Keywords: elliptic operators; VMO-coefficients; weighted Sobolev spaces; maximum principle

\section{Introduction}

It is well known that a priori estimates and uniqueness results, which are necessary in the proof of the well-posedness for boundary value problems for elliptic equations in nondivergence form, are based on Aleksandrov type estimates, i.e., on estimates for the maximum of a solution in terms of the $L^{n}$-norm of the right-hand side.

If $\Omega$ is a bounded domain in $\mathbb{R}^{n}(n>2)$ and

$$
L=\sum_{i, j=1}^{n} a_{i j} \frac{\partial^{2}}{\partial x_{i} \partial x_{j}}+\sum_{i=1}^{n} a_{i} \frac{\partial}{\partial x_{i}}+a
$$

is a uniformly elliptic operator in $\Omega$, the classical result of AD Aleksandrov states that if $u \in C^{0}(\bar{\Omega}) \cap W^{2, n}(\Omega)$, with $u \leq 0$ in $\partial \Omega$, verifies $L u \geq f$, where $a_{i}, a, f \in L^{n}(\Omega)(a \leq 0)$, then

$$
\sup _{\Omega} u \leq c\|f\|_{L^{n}(\Omega)}
$$

where $c \in \mathbb{R}_{+}$depends only on $n, \Omega,\left\|a_{i}\right\|_{L^{n}(\Omega)}$ and on the ellipticity constant.

There have been various directions of developments and extensions of Aleksandrov estimate. For example, maximum principles have been established in different types of boundary problems, such as in the stationary oblique derivative problem or in the stationary Venttsel' problem. Another direction of development of the Aleksandrov ideas is the extension of maximum estimates to equations with lower order coefficients and righthand sides in other function classes (for example, in spaces with anisotropic norms or weighted spaces). In particular, a large number of works is devoted to the weakening of requirements for the right-hand side of the equation considered (see, for example, [1] and its large bibliography).

\section{Springer}

๑2014Caso and D'Ambrosio; licensee Springer. This is an Open Access article distributed under the terms of the Creative Commons Attribution License (http://creativecommons.org/licenses/by/2.0), which permits unrestricted use, distribution, and reproduction in any medium, provided the original work is properly cited. 
In this framework, it is well known that additional hypotheses on the leading coefficients are necessary to obtain the estimates. Several authors have obtained estimates for the maximum of a solution through the $L^{p}$-norms of the right-hand side $(p>n / 2)$ under different conditions on the leading coefficients.

For instance, if $\Omega$ is an arbitrary open subset of $\mathbb{R}^{n}$ and $\left.p \in\right] n / 2,+\infty$ [, a bound of type (1.2) and a consequent uniqueness result can be found in [2]. In fact, it has been proved that, if the coefficients $a_{i j}$ are bounded and locally VMO, the coefficients $a_{i}, a$ satisfy suit-

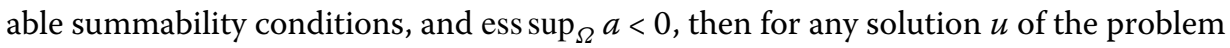

$$
\left\{\begin{array}{l}
u \in W_{\mathrm{loc}}^{2, p}(\Omega) \cap C^{0}(\bar{\Omega}), \\
L u \geq f, \quad f \in L_{\mathrm{loc}}^{p}(\Omega), \\
u_{\mid \partial \Omega} \leq 0 \\
\limsup _{|x| \rightarrow+\infty} u(x) \leq 0 \quad \text { if } \Omega \text { is unbounded, }
\end{array}\right.
$$

there exist a ball $B \subset \subset \Omega$ and a constant $c \in \mathbb{R}_{+}$such that

$$
\sup _{\Omega} u \leq c\left(f_{B}\left|f^{-}\right|^{p} d x\right)^{\frac{1}{p}}
$$

where $f^{-}$is the negative part of $f$,

$$
f_{B}\left|f^{-}\right|^{p} d x=\frac{1}{|B|} \int_{B}\left|f^{-}\right|^{p} d x
$$

and $c$ depends on $n, p$, on the ellipticity constant and on the regularity of the coefficients of $L$.

If the boundary of a domain has various singularities, as for example corners or edges, then, in accordance with the linear theory, it is natural to assume that the lower order coefficients and the right-hand side of the equation belong to some weighted spaces $L^{p}$, where the weight is usually a power of the distance function from the 'singular set' on the boundary of domain. In these cases, the estimates on the solutions are obtained in terms of such weight function.

For instance, if $\rho$ is a bounded weight function related to the distance function from a non-empty subset $S_{\rho}$ of the boundary of an arbitrary domain $\Omega$, not necessarily bounded and regular (see Section 2 for the definition of such weight function), in [3] has been studied a problem similar to the problem (1.3) with boundary conditions and data related to the weight function $\rho$. In particular, if $s \in \mathbb{R}, S_{\rho}=\partial \Omega$, the coefficients $a_{i j}$ are bounded and locally VMO, the coefficients $a_{i}, a$ belong to suitable weighted spaces $L^{\infty}$, in [3] the author has proved that the solution $u$ of the problem

$$
\left\{\begin{array}{l}
u \in W_{\mathrm{loc}}^{2, p}(\Omega), \quad f \in L_{\mathrm{loc}}^{p}(\Omega), \\
L u \geq f, \quad \forall x_{o} \in \partial \Omega, \\
\limsup _{x \rightarrow x_{o}} \rho^{s}(x) u(x) \leq 0, \quad \text { if } \Omega \text { is unbounded, }
\end{array}\right.
$$

verifies the estimate

$$
\sup _{x \in \Omega} \rho^{s}(x) u(x) \leq c\left(f_{B}\left|\rho^{s+2} f^{-}\right|^{p} d x\right)^{\frac{1}{p}},
$$


where $B \subset \subset \Omega$ is an open ball and the constant $c \in \mathbb{R}_{+}$depends on $n, p, s, \rho$, on the ellipticity constant and on the regularity of the coefficients of $L$. As a consequence, some uniqueness results are also obtained. Results of this type are also established in [4] under the more general hypothesis $\emptyset \neq S_{\rho} \subset \partial \Omega$, but for an operator $L$ with coefficients $a_{i}=0$.

The aim of this paper is to improve the above quoted results in [3] by obtaining a similar estimate under much weaker assumptions. In particular, the main difference lies in the hypotheses on the coefficients $a_{i}$, $a$ which are not supposed to belong to weighted spaces $L^{\infty}$ but just to appropriate weighted Sobolev spaces $K_{t}^{r}(\Omega)$ (see Section 2 for the definition of such weighted spaces), which strictly contain the weighted spaces $L^{\infty}$. Moreover, as in [4], we consider the more general hypothesis $\emptyset \neq S_{\rho} \subset \partial \Omega$.

\section{Notation}

In this section we introduce some notation used throughout this paper. Moreover, we recall the definitions of a class of weight functions and of some function spaces in which the coefficients of our operator will be chosen.

Let $A$ be a Lebesgue measurable subset of $\mathbb{R}^{n}$ and let $\Sigma(A)$ be the collection of all Lebesgue measurable subsets of $A$. If $F \in \Sigma(A)$, we denote by $|F|$ the Lebesgue measure of $F$ and by $\mathfrak{D}(F)$ the class of restrictions to $F$ of functions $\zeta \in C_{0}^{\infty}\left(\mathbb{R}^{n}\right)$ with $\bar{F} \cap \operatorname{supp} \zeta \subseteq F$. Moreover, if $X(F)$ is a space of functions defined on $F$, we denote by $X_{\text {loc }}(F)$ the class of all functions $g: F \rightarrow \mathbb{R}$ such that $\zeta g \in X(F)$ for all $\zeta \in \mathfrak{D}(F)$. Furthermore, for $g \in L^{p}(A)$ $(p \geq 1)$, we put

$$
\omega^{p}[g, A](t)=\sup _{\substack{E \in \Sigma(A) \\|E| \leq t}}\|g\|_{L^{p}(E)}, \quad t \in \mathbb{R}_{+} .
$$

Since $\omega^{p}[g, A](t)$ is a decreasing function and $\lim _{t \rightarrow 0} \omega^{p}[g, A](t)=0$, we can refer to $\omega^{p}[g, A]$ as the modulus of continuity of $g$ in $L^{p}(A)$.

Let $\Omega$ be an open subset of $\mathbb{R}^{n}, n \geq 2$. We denote by $\mathcal{A}(\Omega)$ the class of measurable weight functions $\rho: \Omega \rightarrow \mathbb{R}_{+}$such that

$$
\gamma^{-1} \rho(y) \leq \rho(x) \leq \gamma \rho(y), \quad \forall y \in \Omega, \forall x \in \Omega \cap B(y, \rho(y))
$$

where $\gamma \in \mathbb{R}_{+}$is independent of $x$ and $y$, and $B(y, \rho(y))$ is the open ball of radius $\rho(y)$ centered at $y$.

We remark that $\mathcal{A}(\Omega)$ contains the class of all functions $\rho: \Omega \rightarrow \mathbb{R}_{+}$which are Lipschitz continuous in $\Omega$ with Lipschitz constant less than 1 .

Typical examples of functions $\rho \in \mathcal{A}(\Omega)$ are the function

$$
x \in \Omega \rightarrow 1+a|x|, \quad a \in] 0,1[
$$

if $\Omega=\mathbb{R}^{n}$ and, if $\Omega \neq \mathbb{R}^{n}$ and $S$ is a nonempty subset of $\partial \Omega$, the function

$$
x \in \Omega \rightarrow a \cdot \operatorname{dist}(x, S), \quad a \in] 0,1[.
$$

For any $\rho \in \mathcal{A}(\Omega)$ we put

$$
S_{\rho}=\{z \in \partial \Omega|\rho(x) \leq| x-z \mid \forall x \in \Omega\} .
$$


We recall that the set $S_{\rho}$ is a closed subset of $\partial \Omega$ and

$$
z \in S_{\rho} \Longleftrightarrow \lim _{x \rightarrow z} \rho(x)=0
$$

(see [5]).

It is well known that

$$
\rho \in L_{\mathrm{loc}}^{\infty}(\bar{\Omega}), \quad \rho^{-1} \in L_{\mathrm{loc}}^{\infty}\left(\bar{\Omega} \backslash S_{\rho}\right),
$$

and, if $S_{\rho} \neq \emptyset[5,6]$,

$$
\rho(x) \leq \operatorname{dist}\left(x, S_{\rho}\right), \quad \forall x \in \Omega
$$

Let $\rho \in \mathcal{A}(\Omega)$. For $k \in \mathbb{N}_{0}, 1 \leq p \leq+\infty$ and $s \in \mathbb{R}$, we denote by $W_{s}^{k, p}(\Omega)$ the space of distributions $u$ on $\Omega$ such that $\rho^{s+|\alpha|-k} \partial^{\alpha} u \in L^{p}(\Omega)$ for $|\alpha| \leq k$. We observe that $W_{s}^{k, p}(\Omega)$ is a Banach space with the norm defined by

$$
\|u\|_{W_{s}^{k, p}(\Omega)}=\sum_{|\alpha| \leq k}\left\|\rho^{s+|\alpha|-k} \partial^{\alpha} u\right\|_{L^{p}(\Omega)} .
$$

Moreover, it is separable if $1 \leq p<+\infty$, reflexive if $1<p<+\infty$, and, in particular, $W_{s}^{k, 2}(\Omega)$ is an Hilbert space. We put $W_{s}^{0, p}(\Omega)=L_{s}^{p}(\Omega)$, and we observe that the space $C_{0}^{\infty}(\Omega)$ is dense in $L_{s}^{p}(\Omega)$ (see $[7,8]$ ).

A more detailed account of properties of the above defined weighted Sobolev spaces can be found in [7, 9] and [8].

For any $x \in \Omega$, we put

$$
\Omega(x)=\Omega \cap B(x, \rho(x)) .
$$

Let $\rho \in \mathcal{A}(\Omega)$. For $1 \leq p<+\infty$ and $s \in \mathbb{R}$, we denote by $K_{s}^{p}(\Omega)$ the class of functions $g \in$ $L_{\text {loc }}^{p}\left(\bar{\Omega} \backslash S_{\rho}\right)$ such that

$$
\|g\|_{K_{S}^{p}(\Omega)}=\sup _{x \in \Omega}\left(\rho^{s-\frac{n}{p}}(x)\|g\|_{L^{p}(\Omega(x))}\right)<+\infty
$$

Obviously $K_{s}^{p}(\Omega)$ is a Banach space with the norm defined by (2.6). It is easy to prove that the space $L_{s}^{\infty}(\Omega)$ is a subset of $K_{s}^{p}(\Omega)$ (see [10]). Thus, we can define a new space of functions $\widetilde{K}_{s}^{p}(\Omega)$ as the closure of $L_{s}^{\infty}(\Omega)$ in $K_{s}^{p}(\Omega)$.

We recall the following characterization of the above defined space (see [10]):

$$
g \in \widetilde{K}_{s}^{p}(\Omega) \Longleftrightarrow g \in K_{s}^{p}(\Omega) \quad \text { and } \quad \lim _{t \rightarrow 0}\left(\sup _{\substack{E \in \Sigma(\Omega) \\ \sup _{x \in \Omega} \frac{|\Omega(x) \cap E|}{\rho^{n}(x)} \leq t}}\left\|g \chi_{E}\right\|_{K_{s}^{p}(\Omega)}\right)=0
$$

where $\chi_{E}$ denotes the characteristic function of the set $E$. 
Therefore, we define modulus of continuity of $g$ in $\widetilde{K}_{s}^{p}(\Omega)$ as a map $\widetilde{\omega}_{s}^{p}[g]: \mathbb{R}_{+} \rightarrow \mathbb{R}_{+}$such that [11]

$$
\begin{aligned}
& \sup _{\substack{E \in \Sigma(\Omega) \\
\sup _{x \in \Omega} \frac{|\Omega(x) \cap E|}{\rho^{n}(x)} \leq t}}\left\|g \chi_{E}\right\|_{K_{s}^{p}(\Omega)} \leq \widetilde{\omega}_{s}^{p}[g](t), \\
& \lim _{t \rightarrow 0} \widetilde{\omega}_{s}^{p}[g](t)=0 .
\end{aligned}
$$

Further properties of above mentioned function spaces can be found in [5, 10], and [11]. If $\Omega$ has the property

$$
\left.\left.|\Omega(x, r)| \geq A r^{n}, \quad \forall x \in \Omega, \forall r \in\right] 0,1\right]
$$

where $\Omega(x, r)=B(x, r) \cap \Omega$ and $A$ is a positive constant independent of $x$ and $r$, it is possible to consider the space $B M O(\Omega, t)\left(t \in R_{+}\right)$composed by all functions $g \in L_{\text {loc }}^{1}(\bar{\Omega})$ such that

$$
[g]_{B M O(\Omega, t)}=\sup _{\substack{x \in \Omega \\ r \in] 0, t]}} f_{\Omega(x, r)}\left|g-f_{\Omega(x, r)} g\right|<+\infty,
$$

where

$$
f_{\Omega(x, r)} g=|\Omega(x, r)|^{-1} \int_{\Omega(x, r)} g .
$$

If $g \in B M O(\Omega)=B M O\left(\Omega, t_{A}\right)$, with

$$
t_{A}=\sup _{t \in \mathbb{R}_{+}}\left(\sup _{\substack{x \in \Omega \\ r \in] 0, t]}} \frac{r^{n}}{|\Omega(x, r)|} \leq \frac{1}{A}\right),
$$

we will say that $g \in V M O(\Omega)$ if $[g]_{B M O(\Omega, t)} \rightarrow 0$ for $t \rightarrow 0^{+}$. A function $\eta[g]: \mathbb{R}_{+} \rightarrow \mathbb{R}_{+}$is called a modulus of continuity of $g$ in $V M O(\Omega)$ if

$$
[g]_{B M O(\Omega, t)} \leq \eta[g](t), \quad \forall t \in \mathbb{R}_{+}, \quad \lim _{t \rightarrow 0^{+}} \eta[g](t)=0 .
$$

We say that $g \in V M O_{\text {loc }}(\Omega)$ if $(\zeta g)_{o} \in V M O\left(\mathbb{R}^{n}\right)$ for any $\zeta \in C_{0}^{\infty}(\Omega)$, where $(\zeta g)_{o}$ denotes the zero extension of $\zeta g$ outside of $\Omega$. A more detailed account of properties of the above defined spaces $B M O(\Omega)$ and $V M O(\Omega)$ can be found in [12].

We conclude this section introducing a class of applications needed in the sequel.

From now on we consider $\rho \in \mathcal{A}(\Omega) \cap L^{\infty}(\Omega)$ and we suppose that the following condition on $\rho$ holds:

( $\mathrm{h}_{0}$ ) there exists a function $\sigma \in \mathcal{A}(\Omega) \cap C^{\infty}(\Omega) \cap C^{0,1}(\bar{\Omega})$ which is equivalent to $\rho$ and such that

$$
\left|\partial^{\alpha} \sigma(x)\right| \leq c_{\alpha} \sigma^{1-|\alpha|}(x), \quad \forall x \in \Omega, \forall \alpha \in \mathbb{N}_{0}^{n},
$$

where $c_{\alpha}$ is independent of $x$ (see [6]). 
We observe that the condition $\left(\mathrm{h}_{0}\right)$ holds, for example, if $\Omega$ is an unbounded open set with the cone property, or if the open set $\Omega$ has not the cone property but the weight function $\rho$ is equivalent to the function $\operatorname{dist}(\cdot, \partial \Omega)$ (see [6]).

Let us fix $g \in C_{0}^{\infty}\left(\overline{\mathbb{R}}_{+}\right)$satisfying the conditions

$$
0 \leq g \leq 1, \quad g(t)=1 \quad \text { if } t \geq 1, \quad g(t)=0 \quad \text { if } t \leq \frac{1}{2} .
$$

For each $k \in \mathbb{N}$, we put

$$
\eta_{k}(x)=\frac{1}{k} \zeta_{k}(x)+\left(1-\zeta_{k}(x)\right) \cdot \sigma(x), \quad x \in \Omega,
$$

where $\zeta_{k}(x)=g(k \sigma(x)), x \in \Omega$. Obviously, $\eta_{k} \in C^{\infty}(\Omega)$ for any $k \in \mathbb{N}$ and

$$
\eta_{k}(x)= \begin{cases}\frac{1}{k} & \text { if } x \in \bar{\Omega}_{k}, \\ \sigma(x) & \text { if } x \in \Omega \backslash \Omega_{2 k},\end{cases}
$$

where

$$
\Omega_{k}=\left\{x \in \Omega \mid \sigma(x)>\frac{1}{k}\right\} .
$$

Moreover, for $k \in \mathbb{N}$, it is easy to prove that

$$
\begin{aligned}
& \sigma(x) \leq \eta_{k}(x) \leq 2 \sigma(x), \quad x \in \Omega \backslash \bar{\Omega}_{k}, \\
& c^{\prime}{ }_{k} \sigma(x) \leq \eta_{k}(x) \leq \sigma(x), \quad x \in \Omega_{k}, \\
& \left(\eta_{k}(x)\right)_{x} \leq c_{1}(\sigma(x))_{x}, \quad x \in \Omega, \\
& \left(\eta_{k}(x)\right)_{x x} \leq c_{2} \frac{(\sigma(x))_{x}^{2}+\sigma(x) \cdot(\sigma(x))_{x x}}{\sigma(x)}, \quad x \in \Omega,
\end{aligned}
$$

where $c^{\prime}{ }_{k} \in \mathbb{R}_{+}$depends on $k$ and $\sigma$, and $c_{1}, c_{2} \in \mathbb{R}_{+}$depend only on $n$. Furthermore, for any $s \in \mathbb{R}$, we have

$$
\begin{aligned}
& \frac{\left(\eta_{k}^{s}(x)\right)_{x}}{\eta_{k}^{s}(x)} \leq c_{3} \frac{\left(\eta_{k}(x)\right)_{x}}{\sigma(x)}, \quad x \in \Omega, \\
& \frac{\left(\eta_{k}^{s}(x)\right)_{x x}}{\eta_{k}^{s}(x)} \leq c_{3} \frac{\left(\eta_{k}(x)\right)_{x}^{2}+\eta_{k}(x) \cdot\left(\eta_{k}(x)\right)_{x x}}{\sigma^{2}(x)}, \quad x \in \Omega,
\end{aligned}
$$

where $c_{3} \in \mathbb{R}_{+}$depends on $s$ and $n$.

\section{Hypotheses and preliminary results}

Suppose that $\Omega$ has the property (2.8) and let $p>n / 2$. Consider in $\Omega$ the differential operator $\widetilde{L}$ defined by

$$
\widetilde{L}=\sum_{i, j=1}^{n} a_{i j} \frac{\partial^{2}}{\partial x_{i} \partial x_{j}}+\sum_{i=1}^{n} d_{i} \frac{\partial}{\partial x_{i}}+d,
$$


with the following assumptions on the coefficients:

$\left(\mathrm{h}_{1}\right) \quad\left\{\begin{array}{l}a_{i j}=a_{j i} \in L^{\infty}(\Omega) \cap V M O_{\mathrm{loc}}(\Omega), \quad i, j=1, \ldots, n, \\ \exists v_{o}, v \in \mathbb{R}_{+}: \sum_{i, j=1}^{n}\left\|a_{i j}\right\|_{L^{\infty}(\Omega)} \leq v_{o}, \\ \sum_{i, j=1}^{n} \xi_{i} \xi_{j} a_{i j} \geq v|\xi|^{2} \quad \text { a.e. in } \Omega, \forall \xi \in \mathbb{R}^{n},\end{array}\right.$
$\left(\mathrm{i}_{1}\right) \quad\left\{\begin{array}{l}d_{i}=a_{i}+\widetilde{d}_{i}, \quad a_{i} \in \widetilde{K}_{1}^{r}(\Omega), \widetilde{d}_{i} \in L_{1}^{\infty}(\Omega), i=1, \ldots, n, \\ d=a+\widetilde{d}, \quad a \in \widetilde{K}_{2}^{p}(\Omega), \tilde{d} \in L_{2}^{\infty}(\Omega), \\ d \leq 0 \text { a.e. in } \Omega,\end{array}\right.$

where $r>n$ if $p \leq n$, and $r=p$ if $p>n$.

Fixing $x_{o} \in \Omega$ and $\tau \in \mathbb{R}_{+}$such that $\tau \leq \sigma\left(x_{o}\right)$, we put $B=B\left(x_{o}, \tau\right)$ and $B^{*}=B\left(x_{o}, 1\right)$.

We observe that under assumptions $\left(\mathrm{h}_{1}\right)$ and $\left(\mathrm{i}_{1}\right)$, the operator $\widetilde{L}$ from $W^{2, p}(B)$ into $L^{p}(B)$ is bounded and the following estimate holds:

$$
\|\widetilde{L} u\|_{L^{p}(B)} \leq c^{\prime}\|u\|_{W^{2, p}(B)}, \quad \forall u \in W^{2, p}(B)
$$

where $c^{\prime} \in \mathbb{R}_{+}$depends on $n, p, r, \rho, v_{o},\left\|a_{i}\right\|_{L^{r}(B)},\left\|\widetilde{d}_{i}\right\|_{L^{\infty}(B)},\|a\|_{L^{p}(B)},\|\widetilde{d}\|_{L^{\infty}(B)}$.

Let $v$ be a solution of the problem

$$
\left\{\begin{array}{l}
v \in W^{2, p}(B), \\
\widetilde{L} v \geq h, \quad h \in L^{p}(B), \\
v_{\text {|əB }} \leq 0 .
\end{array}\right.
$$

We want to prove a bound for the solution $v$ of the above problem (see Lemma 3.1 below), which will be the primary technical tool in the proof of our main result (see the next Section). In order to use a classical result of Vitanza (see, Theorem 2.1 in [13]) it is necessary to make an appropriate change of variables which allows to transform the operator $\widetilde{L}$ into a differential operator $\widetilde{L}^{*}$ whose lower order coefficients, in particular, belonging to Lebesgue spaces and their moduli of continuity can be estimated by moduli of continuity of the corresponding coefficients of $\widetilde{L}$. To this aim, let us consider the map $T: B \rightarrow B^{*}$ defined by

$$
T(x)=x_{o}+\frac{x-x_{o}}{\tau} .
$$

Clearly

$$
z=T(x) \quad \Leftrightarrow \quad x=x_{o}+\tau\left(z-x_{o}\right)=T^{-1}(z) .
$$

For any function $g$ defined on $B$, we set

$$
g^{*}=g \circ T^{-1} .
$$

Using the equivalence between $\rho$ and $\sigma$ it is easy to prove that $\tau d_{i}^{*} \in L^{r}\left(B^{*}\right)$ for any $i=$ $1, \ldots, n$ and $\tau^{2} d^{*} \in L^{p}\left(B^{*}\right)$; moreover,

$$
\left\|\tau d_{i}^{*}\right\|_{L^{r}\left(B^{*}\right)} \leq c_{1}\left(\left\|a_{i}\right\|_{K_{1}^{r}(\Omega)}+\left\|\tilde{d}_{i}\right\|_{L_{1}^{\infty}(\Omega)}^{\frac{1}{r}}\right), \quad i=1, \ldots, n,
$$


and

$$
\left\|\tau^{2} d^{*}\right\|_{L^{p}\left(B^{*}\right)} \leq c_{2}\left(\|a\|_{K_{2}^{p}(\Omega)}+\|\tilde{d}\|_{L_{2}^{\infty}(\Omega)}^{\frac{1}{p}}\right)
$$

where $c_{1} \in \mathbb{R}_{+}$depends on $n, \rho, r$ and $c_{2} \in \mathbb{R}_{+}$depends on $n, \rho, p$.

On the other hand, for any $E^{*} \in \Sigma\left(B^{*}\right)$ and $t \in \mathbb{R}_{+}$, we have $\left|E^{*}\right| \leq t$ if and only if $\frac{|E|}{\tau^{n}} \leq t$ where $E=\left\{x \in B \mid x=T^{-1}(z), z \in E^{*}\right\}$. Thus, we obtain

$$
\begin{aligned}
& \omega^{r}\left[\tau d_{i}^{*}, B^{*}\right](t) \leq \sup _{\substack{E \in \Sigma(B) \\
\frac{|E|}{\sigma^{n}\left(x_{0}\right)} \leq t}}\left\|\tau^{1-\frac{n}{r}} d_{i}\right\|_{L^{r}(E)}, \quad i=1, \ldots, n, \\
& \omega^{p}\left[\tau^{2} d^{*}, B^{*}\right](t) \leq \sup _{\substack{E \in \Sigma(B) \\
\frac{|E|}{\sigma^{n}\left(x_{0}\right)} \leq t}}\left\|\tau^{2-\frac{n}{p}} d\right\|_{L^{p}(E)} .
\end{aligned}
$$

Using again the equivalence between $\rho$ and $\sigma$ and (2.7), from (3.6) we also deduce

$$
\omega^{r}\left[\tau d_{i}^{*}, B^{*}\right](t) \leq c_{3}\left(\widetilde{\omega}_{1}^{r}\left[a_{i}\right](t)+\left\|\tilde{d}_{i}\right\|_{L_{1}^{\infty}(\Omega)}^{\frac{1}{r}} \cdot t^{\frac{1}{r}}\right), \quad i=1, \ldots, n
$$

and

$$
\omega^{p}\left[\tau^{2} d^{*}, B^{*}\right](t) \leq c_{4}\left(\widetilde{\omega}_{2}^{p}[a](t)+\|\widetilde{d}\|_{L_{2}^{\infty}(\Omega)}^{\frac{1}{p}} \cdot t^{\frac{1}{p}}\right)
$$

where $c_{3} \in \mathbb{R}_{+}$depends on $\rho, r$ and $c_{4} \in \mathbb{R}_{+}$depends on $\rho, p$.

We are now able to prove the requested $a$ priori bound.

Lemma 3.1 Suppose that the conditions $\left(\mathrm{h}_{1}\right)$ and $\left(\mathrm{i}_{1}\right)$ hold. Let $v$ be a solution of the problem (3.1). Then there exists $c_{o} \in \mathbb{R}_{+}$such that

$$
\sup _{B} v \leq c_{o} \cdot \tau^{2-\frac{n}{p}}\left\|h^{-}\right\|_{L^{p}(B)},
$$

where $c_{o}$ depends on $n, p, r, \rho, v, v_{0},\left[p\left(a_{i j}\right)\right]_{B M O\left(\mathbb{R}^{n}, \cdot\right)},\left\|a_{i}\right\|_{K_{1}^{r}(\Omega)},\|a\|_{K_{2}^{p}(\Omega)},\left\|\tilde{d}_{i}\right\|_{L_{1}^{\infty}(\Omega)}$, $\|\widetilde{d}\|_{L_{2}^{\infty}(\Omega)}, \widetilde{\omega}_{1}^{r}\left[a_{i}\right], \widetilde{\omega}_{2}^{p}[a]$, and where $p\left(a_{i j}\right)$ are the extensions of $a_{i j}$ to $\mathbb{R}^{n}$ in $L^{\infty}\left(\mathbb{R}^{n}\right) \cap$ $\operatorname{VMO}\left(\mathbb{R}^{n}\right)$ for any $i, j=1, \ldots, n$.

Proof Let $v \in W^{2, p}(B)$. Taking into account the definitions (3.2) and (3.3), it is easily seen that

$$
\begin{aligned}
(\widetilde{L} v)^{*} & =\sum_{i, j=1}^{n} a_{i j}^{*}\left(v_{x_{i} x_{j}}\right)^{*}+\sum_{i=1}^{n} d_{i}^{*}\left(v_{x_{i}}\right)^{*}+d^{*} v^{*} \\
& =\tau^{-2} \sum_{i, j=1}^{n} a_{i j}^{*} v_{z_{i} z_{j}}^{*}+\tau^{-1} \sum_{i=1}^{n} d_{i}^{*} v_{z_{i}}^{*}+d^{*} v^{*},
\end{aligned}
$$

and hence

$$
\tau^{2}(\widetilde{L} v)^{*}=\widetilde{L}^{*} v^{*}
$$


where

$$
\widetilde{L}^{*}=\sum_{i, j=1}^{n} a_{i j}^{*} \frac{\partial^{2}}{\partial z_{i} \partial z_{j}}+\tau \sum_{i=1}^{n} d_{i}^{*} \frac{\partial}{\partial z_{i}}+\tau^{2} d^{*}
$$

Let us denote by $p\left(a_{i j}\right)$ the extensions of $a_{i j}$ to $\mathbb{R}^{n}$ such that

$$
p\left(a_{i j}\right) \in L^{\infty}\left(\mathbb{R}^{n}\right) \cap \operatorname{VMO}\left(\mathbb{R}^{n}\right), \quad i, j=1, \ldots, n
$$

(for the existence of such functions see Theorem 5.1 in [12]). Since

$$
p\left(a_{i j}\right)^{*} \in L^{\infty}\left(\mathbb{R}^{n}\right) \cap V M O\left(\mathbb{R}^{n}\right), \quad p\left(a_{i j}\right)_{B^{*}}^{*}=a_{i j}^{*}, i, j=1, \ldots, n,
$$

we have

$$
a_{i j}^{*} \in L^{\infty}\left(B^{*}\right) \cap V M O\left(B^{*}\right), \quad i, j=1, \ldots, n .
$$

Moreover, from assumptions $\left(\mathrm{h}_{1}\right),\left(\mathrm{i}_{1}\right)$, and (3.4), (3.5) it follows that

$$
\left\{\begin{array}{l}
a_{i j}^{*}=a_{j i}^{*}, \quad i, j=1, \ldots, n, \\
\sum_{i, j=1}^{n} \xi_{i} \xi_{j} a_{i j}^{*} \geq v|\xi|^{2} \quad \text { a.e. in } B^{*}, \forall \xi \in \mathbb{R}^{n}, \\
\tau d_{i}^{*} \in L^{r}\left(B^{*}\right), \quad i=1, \ldots, n, \quad \tau^{2} d^{*} \in L^{p}\left(B^{*}\right), \quad d^{*} \leq 0 \text { a.e. in } B^{*},
\end{array}\right.
$$

where $r$ and $p$ are as in hypothesis $\left(\mathrm{i}_{1}\right)$.

Consider now the following problem:

$$
\left\{\begin{array}{l}
\widetilde{L}^{*} w=g, \quad g \in L^{p}\left(B^{*}\right) \\
w \in W^{2, p}\left(B^{*}\right) \cap W^{1, p}\left(B^{*}\right)
\end{array}\right.
$$

Putting together (3.11) and (3.13) with Theorem 2.1 of [13] if $n \geq 3$ or with Theorem 3.5 of [14] if $n=2$, it follows that there exists a unique solution $w$ of (3.14) satisfying the estimate

$$
\|w\|_{W^{2, p}\left(B^{*}\right)} \leq K\|g\|_{L^{p}\left(B^{*}\right)},
$$

where $K \in \mathbb{R}_{+}$depends on $n, p, v, v_{0},\left[p\left(a_{i j}\right)^{*}\right]_{B M O\left(\mathbb{R}^{n}, \cdot\right)},\left\|\tau \cdot d_{i}^{*}\right\|_{L^{r}\left(B^{*}\right)},\left\|\tau^{2} \cdot d^{*}\right\|_{L^{p}\left(B^{*}\right)}$, $\omega^{r}\left[\tau d_{i}^{*}, B^{*}\right], \omega^{p}\left[\tau^{2} d^{*}, B^{*}\right]$.

Thus from (3.15) and classical Sobolev embedding theorems (see Lemma 5.15 in [15]) we deduce that there exists $K_{1} \in \mathbb{R}_{+}$, depending on the same parameters as $K$, such that

$$
\max _{\bar{B}^{*}}|w| \leq K_{1}\|g\|_{L^{p}\left(B^{*}\right)}
$$

and hence for each $z \in B^{*}$ there is a function $G(z, \cdot) \in L^{p^{\prime}}\left(B^{*}\right)\left(1 / p+1 / p^{\prime}=1\right)$ such that

$$
w(z)=-\int_{B^{*}} G(z, y) \cdot g(y) d y .
$$


The map $G(z, \cdot)$ is the Green function for the operator $\widetilde{L}^{*}$ in $B^{*}$ and it has the following properties:

$$
\begin{aligned}
& \int_{B^{*}} G(z, y) \cdot \widetilde{g}(y) d y \geq 0, \quad \forall \widetilde{g} \in L^{p}\left(B^{*}\right), \widetilde{g} \geq 0, \\
& \|G(z, \cdot)\|_{L^{p^{\prime}\left(B^{*}\right)}} \leq K_{1} .
\end{aligned}
$$

Setting $g=\widetilde{L}^{*} v^{*}$ in (3.14), we find that the function $w-v^{*}$, belonging to $W^{2, p}\left(B^{*}\right)$, is a solution of the following problem:

$$
\left\{\begin{array}{l}
\widetilde{L}^{*}\left(w-v^{*}\right)=0 \quad \text { in } B^{*}, \\
\left(w-v^{*}\right)_{\left.\right|_{\partial B^{*}}}=-v_{\left.\right|_{\partial B^{*}} ^{*}}^{*} \geq 0 .
\end{array}\right.
$$

Moreover, from (3.12), (3.13) and Lemma 3.1 of [16] (see also Lemma 3.1 of [2] for the case $n \geq 3$ ) it follows that $w-v^{*} \geq 0$ in $B^{*}$. Finally, applying (3.17) with $g=\widetilde{L}^{*} v^{*}=\tau^{2}(\widetilde{L} v)^{*}$ and using (3.18) and (3.19) we obtain

$$
\begin{aligned}
v^{*}(z) & \leq-\int_{B^{*}} G(z, y) \cdot \tau^{2}(\widetilde{L} v)^{*}(y) d y \\
& \leq-\tau^{2} \int_{B^{*}} G(z, y) \cdot h^{*}(y) d y \leq-2 \tau^{2} \int_{B^{*}} G(z, y) \cdot\left(h^{*}\right)^{-}(y) d y \\
& \leq 2 \tau^{2}\|G(z, \cdot)\|_{L^{p^{\prime}\left(B^{*}\right)}} \cdot\left\|\left(h^{*}\right)^{-}\right\|_{L^{p}\left(B^{*}\right)} \leq 2 \tau^{2} \cdot K_{1}\left\|\left(h^{*}\right)^{-}\right\|_{L^{p}\left(B^{*}\right)}, \quad \forall z \in B^{*} .
\end{aligned}
$$

From (3.21), converting back to the $x$-variables $(z=T(x))$, we easily deduce the estimate (3.9).

\section{Main results}

In this section we use the previous result to prove a bound for the solution of our main problem.

Consider in $\Omega$ the differential operator $L$ defined by

$$
L=\sum_{i, j=1}^{n} a_{i j} \frac{\partial^{2}}{\partial x_{i} \partial x_{j}}+\sum_{i=1}^{n} a_{i} \frac{\partial}{\partial x_{i}}+a,
$$

and put

$$
L_{o}=\sum_{i, j=1}^{n} a_{i j} \frac{\partial^{2}}{\partial x_{i} \partial x_{j}} .
$$

Suppose that the leading coefficients of operator $L$ satisfy the assumption $\left(\mathrm{h}_{1}\right)$ while the lower order coefficients verify the following condition:

$$
\left(\mathrm{h}_{2}\right) \quad\left\{\begin{array}{l}
a_{i} \in \widetilde{K}_{1}^{r}(\Omega), \quad i=1, \ldots, n, \\
a \in \widetilde{K}_{2}^{p}(\Omega), \\
\exists a_{o} \in \mathbb{R}_{+}: \operatorname{ess} \sup _{\Omega} \sigma^{2} a=-a_{o},
\end{array}\right.
$$


where $r$ and $p$ are as in hypothesis $\left(\mathrm{i}_{1}\right)$. Moreover, assume that the following condition on $\rho$ holds:

$$
\left(\mathrm{h}_{3}\right) \lim _{k \rightarrow+\infty}\left(\sup _{\Omega \backslash \Omega_{k}}\left((\sigma(x))_{x}+\sigma(x)(\sigma(x))_{x x}\right)\right)=0,
$$

where $\Omega_{k}$ is defined in (2.10). For an example of function $\rho$ whose regularizing function $\sigma$ satisfy $\left(\mathrm{h}_{3}\right)$ we can refer to [17].

We introduce now a class of mappings needed in the sequel. Let us fix a function $\alpha \in$ $C^{\infty}(\Omega) \cap C^{0,1}(\bar{\Omega})$ which is equivalent to $\operatorname{dist}(\cdot, \partial \Omega$ ) (for more details on the existence of such an $\alpha$ see, for instance, Theorem 2, Chapter IV in [18] and Lemma 3.6.1 in [19]). Hence, for any $m \in \mathbb{N}$ we define the functions

$$
\psi_{m}: x \in \bar{\Omega} \rightarrow g(m \alpha(x))\left(1-g\left(\frac{|x|}{2 m}\right)\right),
$$

where $g \in C^{\infty}\left(\overline{\mathbb{R}}_{+}\right)$verifies (2.9). It is easy to prove that each $\psi_{m}$ belongs to $C_{0}^{\infty}(\Omega)$ and

$$
0 \leq \psi_{m} \leq 1, \quad \operatorname{supp} \psi_{m} \subseteq E_{2 m}, \quad\left(\psi_{m}\right)_{\left.\right|_{\bar{E}_{m}}}=1,
$$

where

$$
E_{m}=\left\{x \in \Omega:|x|<m, \alpha(x)>\frac{1}{m}\right\} .
$$

Remark 4.1 From hypothesis $\left(\mathrm{h}_{1}\right)$ and Lemma 4.2 in [12] it follows that for any $m \in \mathbb{N}$ the functions $\left(\psi_{m} a_{i j}\right)_{o}$ (obtained as extensions of $\psi_{m} a_{i j}$ to $\mathbb{R}^{n}$ with zero values out of $\Omega$ ) belong to $V M O\left(\mathbb{R}^{n}\right)$ and

$$
\left[\left(\psi_{m} a_{i j}\right)_{o}\right]_{B M O\left(\mathbb{R}^{n}, t\right)} \leq\left[\psi_{m} a_{i j}\right]_{B M O(\Omega, t)}
$$

for $t$ small enough.

Now we are able to prove our main result.

Theorem 4.2 Suppose that conditions $\left(\mathrm{h}_{1}\right),\left(\mathrm{h}_{2}\right),\left(\mathrm{h}_{3}\right)$ hold. Fixing $s \in \mathbb{R}$, let $u$ be a solution of the problem

$$
\left\{\begin{array}{l}
u \in W_{\mathrm{loc}}^{2, p}(\Omega), \\
L u \geq f, \quad f \in L_{\mathrm{loc}}^{p}(\Omega), \\
\limsup _{x \rightarrow x_{o}} \sigma^{s}(x) u(x) \leq 0, \quad \forall x_{o} \in \partial \Omega, \\
\limsup _{|x| \rightarrow+\infty} \sigma^{s}(x) u(x) \leq 0 \quad \text { if } \Omega \text { is unbounded. }
\end{array}\right.
$$

Then there exist an open ball $B \subset \subset \Omega$ and a constant $c \in \mathbb{R}_{+}$such that

$$
\sup _{x \in \Omega} \sigma^{s}(x) u(x) \leq c\left(f_{B}\left|\sigma^{s+2} f^{-}\right|^{p} d x\right)^{\frac{1}{p}}
$$

where $c$ depends on $n, p, r, \rho, v, v_{0}, a_{o}, \eta\left[\psi_{m} a_{i j}\right](m \in \mathbb{N}),\left\|a_{i}\right\|_{K_{1}^{r}(\Omega)},\|a\|_{K_{2}^{p}(\Omega)}, \widetilde{\omega}_{1}^{r}\left[a_{i}\right], \widetilde{\omega}_{2}^{p}[a]$. 
Proof Without loss of generality it can be assumed that $\sup _{\Omega} \sigma^{s}(x) u(x)>0$. For any $k \in \mathbb{N}$, we put

$$
w_{k}(x)=\eta_{k}^{s}(x) u(x), \quad x \in \Omega
$$

Thus, from the last two conditions of (4.1) and from (2.11), (2.12) and (4.3) it follows that there exists $y_{k} \in \Omega$ such that $\sup _{\Omega} w_{k}(x)=w_{k}\left(y_{k}\right)$. Moreover, taking into account the classical Sobolev embedding theorem (see Theorem 5.4 in [15]), there exists $R_{k} \in$ ]0, $\operatorname{dist}\left(y_{k}, \partial \Omega\right)\left[\right.$ such that $w_{k}(x)>0$ for all $x \in B\left(y_{k}, R_{k}\right)$.

Let $\lambda, \alpha_{k}, \alpha_{o} \in \mathbb{R}_{+}$, with $\alpha_{o}>1$ (which will be suitably chosen later), such that

$$
\alpha_{k}=\alpha_{o} \sigma\left(y_{k}\right), \quad \lambda \leq 1, \quad \lambda \alpha_{k} \leq \min \left\{R_{k}, \sigma\left(y_{k}\right)\right\} .
$$

For simplicity of notation, for each $k \in \mathbb{N}$, we denote by $B_{k}$ the open ball $B\left(y_{k}, \lambda \alpha_{k}\right)$.

Let us set

$$
\varphi_{k}(x)= \begin{cases}1+\lambda^{2}-\frac{\left|x-y_{k}\right|^{2}}{\alpha_{k}^{2}}, & x \in B_{k}, \\ 1, & x \in \Omega \backslash B_{k} .\end{cases}
$$

It is easily seen that

$$
1 \leq \varphi_{k} \leq 1+\lambda^{2} \leq 2
$$

Moreover, for $x \in B_{k}$

$$
\begin{aligned}
& \left(\varphi_{k}\right)_{x_{i}} \leq \frac{2 \lambda}{\alpha_{k}}, \quad\left(\varphi_{k}\right)_{x_{i}} \cdot\left(\varphi_{k}\right)_{x_{j}} \leq \frac{4 \lambda^{2}}{\alpha_{k}^{2}}, \quad i, j=1, \ldots, n, \\
& \left(\varphi_{k}\right)_{x_{i} x_{j}}=0 \quad \text { if } i \neq j, \quad\left(\varphi_{k}\right)_{x_{i} x_{j}}=-\frac{2}{\alpha_{k}^{2}} \quad \text { if } i=j .
\end{aligned}
$$

Consider now the function $v_{k}$ defined by

$$
v_{k}(x)=\varphi_{k}(x) w_{k}(x)-w_{k}\left(y_{k}\right), \quad x \in B_{k} .
$$

Clearly

$$
\left(v_{k}\right)_{\left.\right|_{\partial B_{k}}}=\left(w_{k}\right)_{\left.\right|_{\partial B_{k}}}-w_{k}\left(y_{k}\right) \leq 0, \quad v_{k}\left(y_{k}\right)=\lambda^{2} w_{k}\left(y_{k}\right) .
$$

The first step of the proof is to show that there exists $k_{o} \in \mathbb{N}$ such that, for any $k \geq k_{o}$, each function $v_{k}$ is a solution of a problem of type (3.1), where the coefficients of associated differential operator verify the assumptions of Lemma 3.1.

For any $k \in \mathbb{N}$, it is easy to prove

$$
\begin{gathered}
L_{o} w_{k}-u L_{o} \eta_{k}^{s}-2 \sum_{i, j=1}^{n} a_{i j}\left(\eta_{k}^{s}\right)_{x_{j}} u_{x_{i}}+\sum_{i=1}^{n} a_{i}\left(\eta_{k}^{s} u\right)_{x_{i}} \\
-u \sum_{i=1}^{n} a_{i}\left(\eta_{k}^{s}\right)_{x_{i}}+a \eta_{k}^{s} u=\eta_{k}^{s} L u, \quad x \in \Omega .
\end{gathered}
$$


Since

$$
\left(\eta_{k}^{s}\right)_{x_{j}} u_{x_{i}}=\left(\eta_{k}^{s} u\right)_{x_{i}} \cdot \frac{\left(\eta_{k}^{s}\right)_{x_{j}}}{\eta_{k}^{s}}-\frac{\left(\eta_{k}^{s}\right)_{x_{i}} \cdot\left(\eta_{k}^{s}\right)_{x_{j}}}{\left(\eta_{k}^{s}\right)^{2}} \cdot\left(\eta_{k}^{s} u\right), \quad i, j=1, \ldots, n,
$$

and $u$ is a solution of problem (4.1), from (4.11) we deduce

$$
L_{o} w_{k}+\sum_{i=1}^{n} b_{i}^{k}\left(w_{k}\right)_{x_{i}}+b^{k} w_{k} \geq g^{k} \quad \text { in } \Omega,
$$

where we have put

$$
\begin{aligned}
b_{i}^{k} & =a_{i}-2 \sum_{j=1}^{n} a_{i j} \frac{\left(\eta_{k}^{s}\right)_{x_{j}}}{\eta_{k}^{s}}, \quad i=1, \ldots, n, \\
b^{k} & =a+2 \sum_{i, j=1}^{n} a_{i j} \frac{\left(\eta_{k}^{s}\right)_{x_{i}}\left(\eta_{k}^{s}\right)_{x_{j}}}{\left(\eta_{k}^{s}\right)^{2}}-\sum_{i, j=1}^{n} a_{i j} \frac{\left(\eta_{k}^{s}\right)_{x_{i} x_{j}}}{\eta_{k}^{s}}, \\
g^{k} & =\eta_{k}^{s} f+w_{k} \sum_{i=1}^{n} a_{i} \frac{\left(\eta_{k}^{s}\right)_{x_{i}}}{\eta_{k}^{s}} .
\end{aligned}
$$

We observe that using the hypotheses $\left(\mathrm{h}_{0}\right),\left(\mathrm{h}_{1}\right),\left(\mathrm{h}_{2}\right)$, the equivalence between $\rho$ and $\sigma$, and (2.11)-(2.16), we easily get

$$
\left\{\begin{array}{l}
\left(a_{i j} \cdot \frac{\left(\eta_{k}^{s}\right) x_{j}}{\eta^{s}}\right) \in L_{1}^{\infty}(\Omega), \quad i, j=1, \ldots, n, \\
a_{i j} \cdot \frac{\left(\eta_{k}^{s}\right) x_{i}\left(\eta_{k}^{s}\right) x_{j}}{\left(\eta_{k}^{s}\right)^{2}}, a_{i j} \cdot \frac{\left(\eta_{k}^{s}\right) x_{i} x_{j}}{\eta_{k}^{s}} \in L_{2}^{\infty}(\Omega), \quad i, j=1, \ldots, n, \\
g^{k} \in L_{\mathrm{loc}}^{p}(\Omega) .
\end{array}\right.
$$

Using now the estimate (4.13), it is easily seen that

$$
\begin{aligned}
& L_{o}\left(\varphi_{k} w_{k}\right)-w_{k} L_{o} \varphi_{k}-2 \sum_{i, j=1}^{n} a_{i j}\left(\varphi_{k}\right)_{x_{j}}\left(w_{k}\right)_{x_{i}} \\
& \quad+\sum_{i=1}^{n} b_{i}^{k}\left(\varphi_{k} w_{k}\right)_{x_{i}}-\sum_{i=1}^{n} b_{i}^{k}\left(\varphi_{k}\right)_{x_{i}} w_{k}+b^{k} \varphi_{k} w_{k} \\
& =\varphi_{k}\left(L_{o} w_{k}+\sum_{i=1}^{n} b_{i}^{k}\left(w_{k}\right)_{x_{i}}+b^{k} w_{k}\right) \geq \varphi_{k} g^{k} \quad \text { in } B_{k} .
\end{aligned}
$$

This last inequality can be rewritten as

$$
\begin{gathered}
L_{o}\left(\varphi_{k} w_{k}\right)+\sum_{i=1}^{n} d_{i}^{k}\left(\varphi_{k} w_{k}\right)_{x_{i}}+d^{k} \varphi_{k} w_{k} \\
\geq \varphi_{k} g^{k}+\sum_{i=1}^{n} b_{i}^{k}\left(\varphi_{k}\right)_{x_{i}} w_{k} \quad \text { in } B_{k}
\end{gathered}
$$


where we have set

$$
\begin{aligned}
& d_{i}^{k}=b_{i}^{k}-2 \sum_{j=1}^{n} a_{i j} \frac{\left(\varphi_{k}\right)_{x_{j}}}{\varphi_{k}}, \quad i=1, \ldots, n, \\
& d^{k}=b^{k}+2 \sum_{i, j=1}^{n} a_{i j} \frac{\left(\varphi_{k}\right)_{x_{i}}\left(\varphi_{k}\right)_{x_{j}}}{\left(\varphi_{k}\right)^{2}}-\sum_{i, j=1}^{n} a_{i j} \frac{\left(\varphi_{k}\right)_{x_{i} x_{j}}}{\varphi_{k}} .
\end{aligned}
$$

Hence, putting together (4.9) with (4.19) we get

$$
L_{o} v_{k}+\sum_{i=1}^{n} d_{i}^{k}\left(v_{k}\right)_{x_{i}}+d^{k} v_{k} \geq h^{k} \quad \text { in } B_{k}
$$

where

$$
h^{k}=\varphi_{k} g^{k}+w_{k} \sum_{i=1}^{n} b_{i}^{k}\left(\varphi_{k}\right)_{x_{i}}-d^{k} w_{k}\left(y_{k}\right)
$$

Observe that using the hypotheses $\left(\mathrm{h}_{1}\right),\left(\mathrm{h}_{2}\right)$, and (4.17), (4.5)-(4.8), it is easy to prove that, for any $k \in \mathbb{N}$, the coefficients $d_{i}^{k}$ (for $i=1, \ldots, n$ ) and $d^{k}$ satisfy the first two conditions of assumption ( $\left.\mathrm{i}_{1}\right)$ and the function $h^{k} \in L^{p}\left(B_{k}\right)$. We show now that, for a suitable choice of the constant $\alpha_{o}$, there exists $k_{o} \in \mathbb{N}$ such that for any $k \geq k_{o}$ the coefficients $d^{k}$ verify also the last condition of $\left(\mathrm{i}_{1}\right)$. To this aim, we firstly observe that using again hypotheses $\left(\mathrm{h}_{1}\right)$, $\left(\mathrm{h}_{2}\right)$, and (2.15), (2.16), from (4.15) we obtain

$$
b^{k} \leq-\frac{a_{o}}{\sigma^{2}}+\frac{c_{1}}{\sigma^{2}}\left[\left(\eta_{k}\right)_{x}^{2}+\eta_{k}\left(\eta_{k}\right)_{x x}\right], \quad \text { a.e. in } \Omega,
$$

where $c_{1} \in \mathbb{R}_{+}$depends on $v_{o}, n$ and $s$.

Thus, from (4.4), (2.11)-(2.14) and hypothesis $\left(\mathrm{h}_{3}\right)$ it follows that there exists $k_{o} \in \mathbb{N}$ such that for any $k \geq k_{o}$ we get

$$
b^{k} \leq-\frac{a_{o}}{2 \sigma^{2}}, \quad \text { a.e. in } \Omega .
$$

Now, for $k \geq k_{o}$, putting together (4.25) with (4.21) and using the assumption $\left(\mathrm{h}_{1}\right)$, the properties (4.6)-(4.8) and (4.4), we obtain

$$
d^{k} \leq-\frac{a_{o}}{2 \sigma^{2}}+\frac{8 v_{o} \lambda^{2}}{\alpha_{k}^{2}}+\frac{2 v_{o}}{\alpha_{k}^{2}} \leq\left[-\frac{a_{o}}{2 \cdot \gamma^{2}}+\frac{10 v_{o}}{\alpha_{o}^{2}}\right] \sigma^{-2}\left(y_{k}\right), \quad \text { a.e. in } B_{k} .
$$

Hence, fixing $\alpha_{o}$ such that

$$
\frac{1}{\alpha_{o}^{2}} \leq \frac{a_{o}}{40 v_{o} \cdot \gamma^{2}}
$$

from (4.26) it follows that for each $k \geq k_{o}$

$$
d^{k} \leq-\frac{a_{o}}{4 \gamma^{4} \sigma^{2}(x)}, \quad \text { a.e. in } B_{k} .
$$


Putting together (4.28) with (4.25) and observing that $d^{k}=b^{k}$ in $\Omega \backslash B_{k}$, we deduce that $d^{k} \leq 0$ a.e. in $\Omega$. The above considerations together with (4.4), (4.9), (4.10), and (4.22) show that for any $k \geq k_{o}$ the problem

$$
\left\{\begin{array}{l}
v_{k} \in W^{2, p}\left(B_{k}\right) \\
L_{o} v_{k}+\sum_{i=1}^{n} d_{i}^{k}\left(v_{k}\right)_{x_{i}}+d^{k} v_{k} \geq h^{k}, \quad h^{k} \in L^{p}\left(B_{k}\right), \\
v_{\left.k\right|_{\partial B_{k}} \leq 0}
\end{array}\right.
$$

satisfy the assumptions of Lemma 3.1. Therefore, there exists a constant $c_{1} \in \mathbb{R}_{+}$depending on $n, p, r, \rho, v, v_{o},\left[p\left(a_{i j}\right)\right]_{B M O\left(\mathbb{R}^{n}, \cdot\right)},\left\|a_{i}\right\|_{K_{1}^{r}(\Omega)},\|a\|_{K_{2}^{p}(\Omega)}, \widetilde{\omega}_{1}^{r}\left[a_{i}\right], \widetilde{\omega}_{2}^{p}[a]$ such that

$$
\sup _{B_{k}} v_{k} \leq c_{1}\left(\lambda \alpha_{k}\right)^{2-\frac{n}{p}}\left\|\left(h^{k}\right)^{-}\right\|_{L^{p}\left(B_{k}\right)} .
$$

By (4.10), the last bound with $x=y_{k}$ becomes

$$
\lambda^{2} w_{k}\left(y_{k}\right) \leq c_{1}\left(\lambda \alpha_{k}\right)^{2-\frac{n}{p}}\left\|\left(h^{k}\right)^{-}\right\|_{L^{p}\left(B_{k}\right)} .
$$

Now, in order to obtain the estimate (4.2), we have to provide a lower bound for the function $h^{k}$ in terms of the data $f$. First of all, we observe that, using the definitions (4.14) and (4.16), we can rewrite (4.23) as

$$
\begin{aligned}
h^{k}= & \varphi_{k} \eta_{k}^{s} f+w_{k} \sum_{i=1}^{n} a_{i}\left(\frac{\left(\eta_{k}^{s}\right)_{x_{i}}}{\eta_{k}^{s}} \cdot \varphi_{k}+\left(\varphi_{k}\right)_{x_{i}}\right) \\
& -2 w_{k} \sum_{i, j=1}^{n} a_{i j} \frac{\left(\eta_{k}^{s}\right)_{x_{j}}}{\eta_{k}^{s}} \cdot\left(\varphi_{k}\right)_{x_{i}}-d^{k} w_{k}\left(y_{k}\right) .
\end{aligned}
$$

On the other hand, by assumption $\left(\mathrm{h}_{1}\right)$, and by (2.15), (4.7), and (4.4) we easily obtain

$$
\left|2 \cdot \sum_{i, j=1}^{n} a_{i j} \frac{\left(\eta_{k}^{s}\right)_{x_{j}}}{\eta_{k}^{s}} \cdot\left(\varphi_{k}\right)_{x_{i}}\right| \leq \frac{c_{2}}{\sigma^{2}(x)} \cdot\left(\eta_{k}\right)_{x}
$$

where $c_{2} \in \mathbb{R}_{+}$depends on $v_{o}, n, s, \rho, a_{o}$. Thus, using (2.13) and hypothesis $\left(\mathrm{h}_{3}\right)$ it follows that there exists $k_{1} \geq k_{o}$, with $k_{1} \in \mathbb{N}$, such that for any $k \geq k_{1}$

$$
\left|2 \cdot \sum_{i, j=1}^{n} a_{i j} \frac{\left(\eta_{k}^{s}\right)_{x_{j}}}{\eta_{k}^{s}} \cdot\left(\varphi_{k}\right)_{x_{i}}\right| \leq \frac{a_{o}}{4 \gamma^{4} \sigma^{2}(x)} .
$$

Putting together (4.34) and (4.28) with (4.32) we obtain

$$
h^{k} \geq \varphi_{k} \eta_{k}^{s} f+w_{k} \sum_{i=1}^{n} a_{i}\left(\frac{\left(\eta_{k}^{s}\right)_{x_{i}}}{\eta_{k}^{s}} \cdot \varphi_{k}+\left(\varphi_{k}\right)_{x_{i}}\right) .
$$

Taking into account (4.35), from (4.31) we get

$$
w_{k}\left(y_{k}\right) \leq c_{1} \lambda^{-\frac{n}{p}} \alpha_{k}^{2-\frac{n}{p}}\left\|\left(\eta_{k}^{s} f\right)^{-}\right\|_{L^{p}\left(B_{k}\right)}+\Psi_{1}^{k}+\Psi_{2}^{k},
$$


where we have put

$$
\Psi_{1}^{k}=c_{1} \lambda^{-\frac{n}{p}} \alpha_{k}^{2-\frac{n}{p}}\left\|w_{k} \sum_{i=1}^{n} a_{i}\left(\varphi_{k}\right)_{x_{i}}\right\|_{L^{p}\left(B_{k}\right)},
$$

and

$$
\Psi_{2}^{k}=c_{1} \lambda^{-\frac{n}{p}} \alpha_{k}^{2-\frac{n}{p}}\left\|w_{k} \sum_{i=1}^{n} a_{i} \frac{\left(\eta_{k}^{s}\right)_{x_{i}}}{\eta_{k}^{s}} \cdot \varphi_{k}\right\|_{L^{p}\left(B_{k}\right)}
$$

To end the proof, we give some upper bounds for the functions $\Psi_{1}^{k}$ and $\Psi_{2}^{k}$ (with $k \geq k_{1}$ ). First of all, observe that using (4.7) and Hölder's inequality in (4.37) we obtain

$$
\Psi_{1}^{k} \leq c_{3} \lambda^{1-\frac{n}{r}} \alpha_{k}^{1-\frac{n}{r}} w_{k}\left(y_{k}\right)\left\|\sum_{i=1}^{n} a_{i}\right\|_{L^{r}\left(B_{k}\right)},
$$

where $c_{3} \in \mathbb{R}_{+}$depends on the same parameters as $c_{1}$. Using now (4.4), the equivalence on $\rho$ and $\sigma$, we get

$$
\Psi_{1}^{k} \leq c_{4} \lambda^{1-\frac{n}{r}} \alpha_{o}^{1-\frac{n}{r}} w_{k}\left(y_{k}\right)\left\|\sum_{i=1}^{n} a_{i}\right\|_{K_{1}^{r}(\Omega)},
$$

where $c_{4} \in \mathbb{R}_{+}$depends on the same parameters as $c_{1}$. If we choose $\lambda$ such that

$$
\lambda^{1-\frac{n}{r}} \leq \frac{1}{4 c_{4} \alpha_{o}^{1-n / r}\left\|\sum_{i=1}^{n} a_{i}\right\|_{K_{1}^{r}(\Omega)}}
$$

from (4.40), for $k \geq k_{1}$, we get

$$
\Psi_{1}^{k} \leq \frac{w_{k}\left(y_{k}\right)}{4}
$$

Arguing similarly we obtain, for each $k \geq k_{1}$, the following bound on the function $\Psi_{2}^{k}$ :

$$
\Psi_{2}^{k} \leq c_{5} \lambda^{-\frac{n}{r}} \alpha_{o}^{2-\frac{n}{r}} w_{k}\left(y_{k}\right)\left\|\sum_{i=1}^{n} a_{i}\right\|_{K_{1}^{r}(\Omega)} \sup _{\Omega \backslash \Omega_{k}}\left(\eta_{k}\right)_{x},
$$

where $c_{5} \in \mathbb{R}_{+}$depends on the same parameters as $c_{1}$ and on $s$. Thus, using again (2.13) and assumption $\left(\mathrm{h}_{3}\right)$, we see that there exists $k_{2} \geq k_{1}$, with $k_{2} \in \mathbb{N}$, such that for $k \geq k_{2}$ we get

$$
\Psi_{2}^{k} \leq \frac{w_{k}\left(y_{k}\right)}{4}
$$

Finally, chosen $k=k_{2}$, putting together (4.42) and (4.44) with (4.36) and using (4.4), (2.11), and (2.12) it follows that

$$
w_{k_{2}}\left(y_{k_{2}}\right) \leq c_{6}\left(\lambda \alpha_{k_{2}}\right)^{-\frac{n}{p}}\left\|\sigma^{2+s} f^{-}\right\|_{L^{p}\left(B_{k_{2}}\right)},
$$


where $c_{6} \in \mathbb{R}_{+}$depends on the same parameters as $c_{1}$ and on $a_{0}$. Taking into account (4.3) and using again (2.11) and (2.12), from (4.45) we get

$$
\sup _{\Omega} \sigma^{s}(x) u(x) \leq c_{7}\left(f_{B_{k_{2}}}\left|\sigma^{2+s} f^{-}\right|^{p}\right)^{1 / p},
$$

where $c_{7} \in \mathbb{R}_{+}$depends on the same parameters as $c_{1}$ and on $a_{0}$.

Finally, if we choose

$$
p\left(a_{\left.i j\right|_{B_{k_{2}}}}\right)=\left(\psi_{m_{o}} a_{i j}\right)_{o}
$$

where $m_{o} \in \mathbb{N}$ is such that $\psi_{\left.m_{o}\right|_{B_{k_{2}}}}=1$, the estimate (4.2) follows from (4.46), (4.47), and Remark 4.1.

\section{Competing interests}

The authors declare that they have no competing interests.

\section{Authors' contributions}

All authors contributed equally to the writing of this paper. All authors read and approved the final manuscript.

Received: 16 January 2014 Accepted: 15 April 2014 Published: 06 May 2014

\section{References}

1. Nazarov, Al: The A. D. Aleksandrov maximum principle. J. Math. Sci. (N.Y.) 142, 2154-2171 (2007)

2. Caso, L, Cavaliere, P, Transirico, M: On the maximum principle for elliptic operators. Math. Inequal. Appl. 7, 405-418 (2004)

3. Caso, L: Bounds for elliptic operators in weighted spaces. J. Inequal. Appl. 2006, 76215 (2006)

4. Caso, L: Bounds for solutions to an elliptic problem in weighted spaces. In: Percorsi incrociati (in ricordo di Vittorio Cafagna), pp. 59-71. Rubettino, Soveria Mannelli (2010)

5. Caso, L, Transirico, M: Some remarks on a class of weight functions. Comment. Math. Univ. Carol. 37, 469-477 (1996)

6. Troisi, M: Su una classe di funzioni peso. Rend. Accad. Naz. Sci. XL Mem. Mat. 10, 141-152 (1986)

7. Edmunds, DE, Evans, WD: Elliptic and degenerate-elliptic operators in unbounded domains. Ann. Sc. Norm. Super. Pisa 2, 591-640 (1973)

8. Troisi, M: Su una classe di spazi di Sobolev con peso. Rend. Accad. Naz. Sci. XL Mem. Mat. 10, 177-189 (1986)

9. Benci, V, Fortunato, D: Weighted Sobolev spaces and the nonlinear Dirichlet problem in unbounded domains. Ann. Mat. Pura Appl. 121, 319-336 (1979)

10. Canale, A, Caso, L, Di Gironimo, P: Weighted norm inequalities on irregular domains. Rend. Accad. Naz. Sci. XL Mem. Mat. 16, 193-209 (1992)

11. Caso, L, D'Ambrosio, R: Weighted spaces and weighted norm inequalities on irregular domains. J. Approx. Theory 167, 42-58 (2013)

12. Transirico, M, Troisi, M, Vitolo, A: BMO spaces on domains of $\mathbb{R}^{n}$. Ric. Mat. 45, 355-378 (1996)

13. Vitanza, C: A new contribution to the $W^{2, p}$-regularity for a class of elliptic second order equations with discontinuous coefficients. Matematiche 48, 287-296 (1993)

14. Cavaliere, P, Transirico, M: The Dirichlet problem for elliptic equations in the plane. Comment. Math. Univ. Carol. 46, 751-758 (2005)

15. Adams, RA: Sobolev Spaces. Academic Press, New York (1975)

16. Cavaliere, P, Transirico, M: A strong maximum principle for linear elliptic operators. Int. J. Pure Appl. Math. 57, 299-311 (2009)

17. Caso, L, Transirico, M: The Dirichlet problem for second order elliptic equations with singular data. Acta Math. Hung. 76, 1-16 (1997)

18. Stein, EM: Singular Integrals and Differentiability Properties of Functions. Princeton University Press, Princeton (1970)

19. Ziemer, WP: Weakly Differentiable Functions. Springer, Berlin (1989)

10.1186/1687-2770-2014-91

Cite this article as: Caso and D'Ambrosio: On the maximum principle for elliptic operators in weighted spaces. Boundary Value Problems 2014, 2014:91 